Original Contribution

\title{
DYNAMIC CORRELATION BETWEEN CORNEAL SENSITIVITY AND SUBJECTIVE SYMPTOMS IN PATIENTS WITH CHRONIC CORNEAL ULCERS
}

\author{
R. Hristova*, A. Oscar, S. Kostova, I. Petkova, Y. Zdravkov \\ Department of Ophthalmology, Medical University-Sofia, University Hospital Alexandrovska, \\ Sofia, Bulgaria
}

\begin{abstract}
Purpose: This study assessed the correlation between corneal sensitivity and subjective symptoms in patients with chronic corneal ulcers before and after treatment. Methods: Corneal sensitivity was measured with Cochet-Bonnet aesthesiometry in twenty patients with mean age of53 years $\mathrm{SD} \pm 20.65$. Impression cytology was used to assess conjunctivalization, inflammation and keratinization. The epithelial integrity and stromal reaction were determined by anterior segment OCT. Treatment included either conservative measures or surgical ocular surface reconstruction. A questionnaire on subjective symptoms was implemented in order to obtain comparable data about their severity and effect on everyday life. Results: Mean corneal aesthesiometry was $143.03 \pm 81.68 \mathrm{~mm} / \mathrm{grms}$ before treatment and $72.48 \pm 72.80 \mathrm{~mm} / \mathrm{grms}$ on follow-up $(\mathrm{p}=0.001)$. An inverse correlation between age and corneal sensitivity was observed before treatment $(\mathrm{p}=0.002)$, but not after $(\mathrm{p}=0.102)$. Anterior segment OCT on follow-up demonstrated epithelialized corneal surface, reduced stromal reaction and scar tissue preventing further melting. All tested subjective symptoms demonstrated significant change after treatment except driving. Spearman's analysis demonstrated a correlation between corneal sensitivity and pain $(\mathrm{p}=0.033)$, hyperemia $(\mathrm{p}=0.009)$ and driving $(\mathrm{p}=0.043)$. The change in corneal sensitivity correlated with tearing reduction $(\mathrm{p}=0.046)$ and prolonged tolerance to phone use $(\mathrm{p}=0.044)$. Conclusions: Cochet-Bonnet aesthesiometry is a useful diagnostic tool for evaluating corneal nerve function and monitoring the effect of treatment.
\end{abstract}

Key words: corneal ulcer, aesthesiometry, questionnaire

\section{INTRODUCTION}

The interaction between the optical elements of the eye and the environment is mediated by the tear film and a surface epithelial layer including two phenotypically different types conjunctival and corneal with a transition at the limbal zone [1]. The limbal location of stem cells with regenerative properties, surrounded by a specialized environment called a niche, is supported by a number of studies [2-4].

Corneal nerves play an important role for ocular surface health, including being an element of the stem cell niche. They originate from the first (ophthalmic) division of the

*Correspondence to: Dr. Rozaliya Hristova, 72A Burel str., Sofia 1408, Bulgaria, +359887973514, alleta@abv.bg, Medical University-Sofia, University Hospital Alexandrovska, Sofia, Bulgaria trigeminal nerve and reach the limbus through the ciliary nerves, forming a circumferential plexus near the corneoscleral junction. Radial branches from the plexus enter the corneal stroma losing their myelin sheaths to help retain corneal transparency. In the cornea, nerves travel anteriorly and branch immediately underneath the epithelium diverging vertically to terminate at the level of the apical cells [5]. Corneal nerves and epithelium maintain a mutually beneficial support mechanism through the secretion of certain substances [6]. Therefore, damage of corneal nerves may lead to epithelial defects, impaired tear reflex with signs of dry eye and reduced blinking, inadequate proliferative and migratory function of the limbal stem cells, creating a vicious cycle resulting in loss of epithelial and stromal corneal integrity. All of these can cause severe visual impairment, 
constant ocular discomfort and reduced quality of life.

Hence, quantitative and qualitative measurements of corneal sensitivity could be used as an important indicator of the ocular surface condition and for monitoring the effect of treatment. Cochet-Bonnet aesthesiometry is a simple, relatively objective measure of tactile corneal sensitivity. The device is hand-held and contains a nylon monofilament with a retractable length from 0.5 to $6 \mathrm{~cm}$. By adjusting the length of the filament and the contact area remaining constant (equal to the diameter of the filament) the pressure needed to induce a response is read from a scale ranging from $11 \mathrm{~mm} / \mathrm{gm}$ to $200 \mathrm{~mm} / \mathrm{gm}^{[7}$. Studies on Cochet-Bonnet aesthesiometry have demonstrated variable corneal sensitivity depending on test site, age, temperature, contact lens wear, etc. [7].

The purpose of this study is to assess the correlation between restoration of corneal sensitivity and subjective symptoms in patients with chronic corneal ulcers before and after conservative treatment and surgical ocular surface reconstruction.

\section{METHODS}

This was a prospective observational study approved by the ethics committee of Medical University Sofia (Resolution for Project №
8348/22.11.2018) conducted between March 2019 and March 2020. Informed consent was obtained from all participants included in the study prior to treatment. All procedures performed in studies involving human participants were in accordance with the ethical standards of the institutional research committee and with the 1964 Helsinki declaration and its later amendments or comparable ethical standards. Statistical analysis of the data was performed using IBM SPSS v23.

\section{Patients}

Twenty patients with chronic corneal ulcers and mean age 53 years $\mathrm{SD} \pm 20.65$ (range 1883) were included. A routine ophthalmic examination including visual acuity, intraocular pressure and biomicroscopy was performed. All patients demonstrated characteristic clinical signs including nonhealing corneal ulcers with thickened round edges and a surrounding zone of epithelial weakness with a duration of more than 3 weeks and a history of consecutive treatment with numerous topical medications with no observable improvement (Figure 1A). Infectious aetiology was ruled out based on negative microbiological cultures and Gram staining of corneal and/or conjunctival samples.
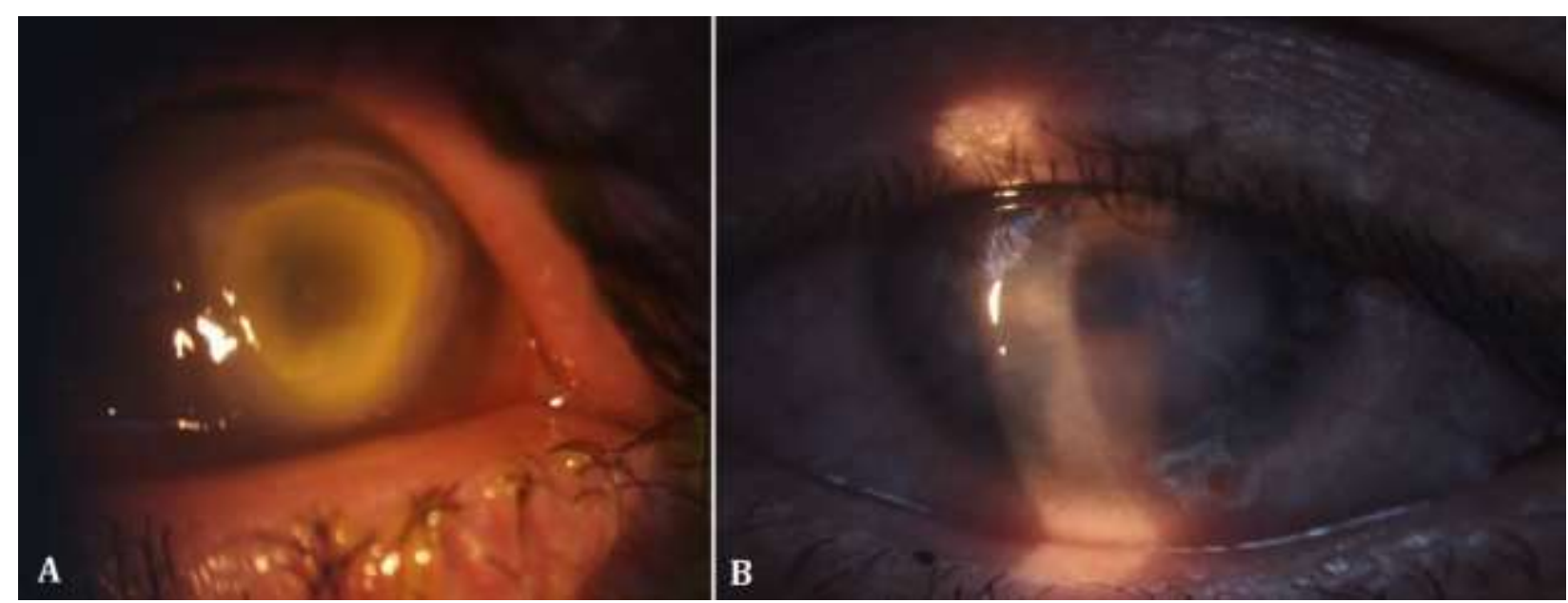

Figure 1. Clinical appearance of a patient with chronic corneal ulcer following endophthalmitis. A. Corneal ulcer with rolled edges and epithelial instability, marked with fluorescein dye. B. On follow-up improved corneal transparency is observed with fine scar tissue.

\section{Aesthesiometry}

Corneal sensitivity threshold was determined by Luneau Cochet-Bonnet aesthesiometry (Western Ophthalmics Corporation, Lynnwood, WA) defined as the length of the nylon filament at which the subject responded to $50 \%$ of the number of stimulations. The measured response as millimetres length of the filament was reported as $\mathrm{mm} / \mathrm{grms}$ using the manufacturer calibration curve. 


\section{Impression cytology}

To study the cellular changes impression cytology samples from all participants were collected using cellulose-acetate membrane filters with pore size 0.4 micrometres by the established technique of Tseng et al [8]. Following instillation of topical anesthetic, impression on the corneal defect with a gentle pressing of the filter, transfer of the sampled cells and fixing the filter in formalin solution. The previously reported method with hematoxylin and eosin, Papanicolau and Periodic acid Schiff was used directly on the filter paper.

\section{Anterior Segment Optical Coherence Tomography (AS-OCT)}

AS-OST was performed prior to and 1 month after treatment to assess the condition using standard protocols Line Anterior Segment, 3D Anterior Segment и Radial Anterior Segment on a spectral domain OCT (3D OCT-2000FA, Topcon). Baseline optical scans were evaluated for the extent of the epithelial irregularities, stromal hyper reflectivity, edema and ulcer depth in the affected eye. After treatment, the state of the epithelium and the change in stromal tissue were characterized.

\section{Treatment}

Treatment included conservative measures comprising of topical artificial tears with vitamins $\mathrm{A}$ and $\mathrm{B}$, autologous serum eye drops, subconjunctival application of steroids as needed and temporary patching of the eyelids. If no improvement was observed for 7 days, surgical reconstruction of the ocular surface was performed with either amniotic membrane or glycerolized cornea transplantation. Postoperatively patients were treated with a fixed combination of prednisolone acetate and tobramycin eye drops four times daily for 1 week with occlusion by sterile patch. After taper for two weeks, treatment was replaced by artificial tears 3 times daily.

\section{Subjective symptoms questionnaire}

A questionnaire based on the Ocular Comfort Index and Quality of Life studies [9] was implemented before and after treatment in order to obtain objective and comparable data about the severity of symptoms and their effect on everyday life. The patients answered questions about symptoms such as discomfort, stinging, tearing, pain, redness and photosensitivity before and after treatment. The medication used was determined as the number of separate bottles of eye drops without taking into account combination products. The everyday activities most likely to be affected by the ocular surface disease were defined as reading, telephone use, time spent in front of an electronic (TV) screen. Activities such as driving, going out to a restaurant, cinema, theatre or sport events are affected not only by ocular symptoms, but by the psychological effect of frequent use of topical medication in public as well as intolerance to air-conditioned spaces.

\section{RESULTS \\ Aesthesiometry}

Mean corneal aesthesiometry before treatment was $143.03 \pm 81.68 \mathrm{~mm} / \mathrm{grms}$. On follow-up mean aesthesiometry was $72.48 \pm 72.80$ $\mathrm{mm} /$ grms. One patient did not show change in corneal sensitivity. Wilcoxon's signed rank test demonstrated the change was statistically significant $(p=0.000)$. Using Spearman's test an inverse correlation between age and corneal sensitivity was observed before treatment (Spearman's rho $=0.653, \mathrm{p}=0.002$ ), but not after (Spearman's rho $=0.376, \mathrm{p}=0.102$ ).

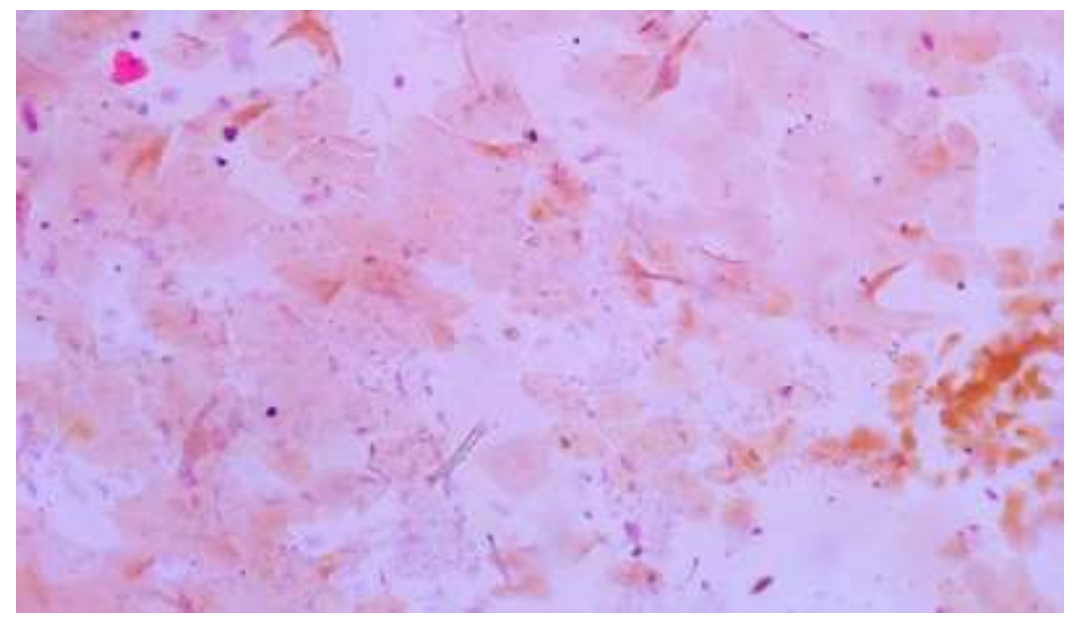

Figure 2. Impression cytology of corneal surface showing keratinization and PAS+ cells. (PAP, PAS, x100) 


\section{Impression cytology}

All patients demonstrated conjunctivalization, presented as PAS+ cells in the corneal epithelium and inflammatory cellular infiltrates consisting primarily of lymphocytes. In cases with deeper ulceration $(n=10)$ the samples directly acquired cells of the basal layers without the need of consecutive impressions. In all cases mild to moderate xerosis with keratinization was observed on Papanicolau stain (Figure 2).

\section{Anterior Segment Optical Coherence Tomography}

Baseline AS-OCT demonstrated extensive damage to the epithelium, moderate to marked stromal hyperreflectivity and oedema with a tendency for confluence progressing to lipid degeneration (Figure 3A). In some of the cases $(n=10)$ stromal melting was advanced with threatening corneal perforation. (Figure 3B). On follow-up 1 month after treatment the corneal surface was completely epithelialized. Stromal hyperreflectivity and oedema were reduced or absent and fine scar tissue with demarcation line had formed, which prevented further melting and improved in transparency during the convalescent period (Figure 3C).
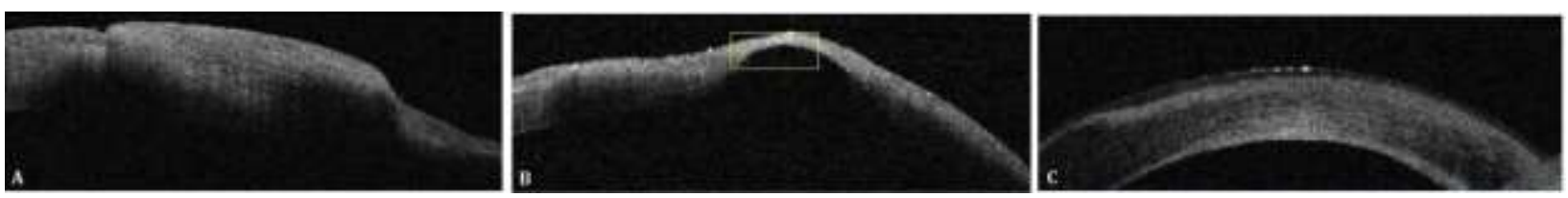

Figure 3. A. Anterior segment OCT of a corneal ulcer with lipid degeneration demonstrating epithelial irregularities, stromal hyperreflectivity and oedema. B. Impending descemetocele. C. Same patient as A after treatment - restored epithelial integrity, reduced stromal hyperreflectivity without oedema, fine scar tissue with demarcation line.

\section{Subjective symptoms questionnaire}

The internal consistency and reliability of the questionnaire were estimated using Cronbach's alpha $(\alpha=0.778)$. The results before and after treatment were compared (Table 1) using Wilcoxon's signed rank test. All tested parameters demonstrated significant change after treatment except driving.

Spearman's analysis demonstrated significant correlation between corneal sensitivity and subjective symptoms before and after treatment including pain (Spearman's rho $=-.338, \mathrm{p}=0.033$ ), hyperemia (Spearman's rho $=-.405, \mathrm{p}=0.009$ ) and driving (Spearman's rho=.322, $\quad \mathrm{p}=0.043)$. The extent of improvement in corneal sensitivity did not correlate with the change in most subjective symptoms except with the reduction in tearing $(\mathrm{p}=0.046)$ and prolonged tolerance to phone use $(\mathrm{p}=0.044)$.

Table 1. Comparison between baseline and follow-up in subjective symptoms questionnaire

\begin{tabular}{|l|l|}
\hline Question & P-value \\
\hline Discomfort, grittiness & 0.001 \\
\hline Tearing & 0.001 \\
\hline Pain & 0.001 \\
\hline Photophobia (sensitivity to light) & 0.002 \\
\hline Hyperemia (redness) & 0.001 \\
\hline Number of topical medication & 0.001 \\
\hline Reading & 0.006 \\
\hline Television/electronic screen & 0.006 \\
\hline Driving & 0.102 \\
\hline Events (theatre, cinema, sports, restaurant) & 0.025 \\
\hline Telephone use & 0.004 \\
\hline
\end{tabular}

\section{DISCUSSION}

Corneal sensitivity is a simple, repeatable and cost-effective method for evaluating corneal nerves integrity. In this study the method was used to assess the effect of treatment on subjective symptoms. At baseline corneal sensitivity decreased with age, which supports previous findings [10]. However, treatment 


\section{HRISTOVA R., et al.}

changed this correlation since individual patients responded with different extent of improvement. Moreover, in one case sensitivity did not change on follow-up which suggests improvement may require a longer time period in more severe ocular surface disease.

Baseline AS-OCT demonstrated extensive damage to the epithelium and marked stromal hyperreflectivity, which is considered to represent activated stromal keratocytes and inflammatory cellular, infiltrates [11]. Fine scar tissue remained after treatment which prevented further melting possibly due to spontaneous collagen crosslinking, which was later remodelled to improve transparency, similar to changes observed after corneal crosslinking for keratoconus [12].

The conjunctivalization of the corneal surface along with chronic inflammation suggests associated limbal stem cell deficiency. This confirms the interdependence between corneal nerve function and epithelial integrity. The observed xerosis with keratinization of the epithelium might be a consequence of dellen formation next to the rolled edge of the ulcer with tear film disturbance and local deficit of vitamin A. Samples from patients with deeper ulcers directly collected cells from the basal layers probably due to increased instability of newly formed epithelium with insufficient tight junctions with the basal membrane.

The results from the subjective symptoms questionnaire show improvement in all tested parameters, except driving, which may be due to personal factors - either not owning a vehicle or not having a driver's license, as well as the more advanced age of some participants. The reduction in used topical medication is of utmost importance in chronic corneal ulcers due to lower cumulative toxic effect of preservatives often contained in each separate preparation [13]. Patients reported increased tolerance to electronic screens and phones, as well as reading, which significantly increases the quality of life. Corneal sensitivity correlated with levels of subjective perception of pain and hyperaemia. Patients with chronic corneal ulcers and especially neurotrophic keratopathy have highly variable pain perception, since aberrant nerve regeneration leads to microneuroma formation [14]. These structures demonstrate abnormal excitability and conductivity, which leads to dysesthesia.
The extent of increase in corneal sensitivity correlated with the extent of tearing reduction and tolerance to phone use, which can be explained by improvement in the conductivity along the tear reflex pathway and increased blink rate. This emphasizes the role of corneal innervation for tear film stability and therefore ocular surface integrity.

\section{CONCLUSION}

Cochet-Bonnet aesthesiometry is a useful diagnostic tool for evaluating corneal nerve function and monitoring the effect of treatment on objective ocular surface convalescence, as well as improvement in subjective symptoms.

\section{ACKNOWLEDGEMENTS}

This work is supported by the Bulgarian Ministry of Education and Science under the National Program for Research "Young Scientists and Postdoctoral Students".

Conflicts of Interest: All authors declare that there is no conflict of interest regarding the publication of this paper.

\section{REFERENCES}

1. Tseng SCG, Tsubota K. Important concepts for treating ocular surface and tear disorders. Am J Opthalmol; 124:825-35, 1997.

2. Dua H.S., Azuara-Blanco A. Limbal stem cells of the corneal epithelium. Survey of Ophthalmology. 44(5):415-425, 2000.

3. Pellegrini G, Rama P, Mavilio F, De Luca M. Epithelial stem cells in corneal regeneration and epidermal gene therapy. $J$ Pathol; 217:217-228, 2009.

4. Schermer A, Galvin S, Sun TT. Differentiation-related expression of a major $64 \mathrm{~K}$ corneal keratin in vivo and in culture. J Cell Biol. 103:49-62, 1986

5. Tasman, W., Jaeger, E.A., Duane's Ophthalmology. LWW, Hagerstown, MD, USA, 2007.

6. Garcia-Hirschfeld J, Lopez-Briones LG, Belmonte C. Neurotrophic influences on corneal epithelial cells. Experimental Eye Research. 59(5):597-605, 1994.

7. Acosta MC, Alfaro ML, Borrás $\mathrm{F}$, Belmonte C, Gallar J. Influence of age, gender and iris color on mechanical and chemical sensitivity of the cornea and conjunctiva. Exp. Eye Res. 83(4):932-938.

8. Tseng SCG. Staging of conjunctival squamous metaplasia by impression 
cytology. Ophthalmology 1985;92:728-33, 2006.

9. Valikodath NG, Newman-Casey PA, Lee PP, Musch DC, Niziol LM, Woodward MA. Agreement of Ocular Symptom Reporting Between Patient-Reported Outcomes and Medical Records. JAMA Ophthalmol. 135(3):225-231, 2017.

10. Millodot M. The influence of age on the sensitivity of the cornea. Invest Ophthalmol Vis Sci. 16:241-244, 1977.

11. Shousha MA, Karp CL, Canto AP, Hodson K, Oellers P, Kao AA, et al. Diagnosis of ocular surface lesions using ultra-high resolution optical coherence tomography. Ophthalmology;120:883-91, 2013.
12.Spadea L, Di Genova L, Tonti E. Corneal stromal demarcation line after 4 protocols of corneal crosslinking in keratoconus determined with anterior segment optical coherence tomography. J Cataract Refract Surg. 44(5):596-602, 2018.

13. Kahook MY, Ammar DA: In vitro toxicity of topical ocular prostaglandin analogs and preservatives on corneal epithelial cells. $J$ Ocul Pharmacol Ther;26:259-263, 2010.

14.Benítez-del-Castillo JM, Acosta CM, Wassfi MA, et al. Relation between Corneal Innervation with Confocal Microscopy and Corneal Sensitivity with Noncontact Esthesiometry in Patients with Dry Eye. Invest. Ophthalmol. Vis. Sci.48(1):173-181, 2007. 